\title{
Bose-Einstein condensates with localized spin-orbit coupling: Soliton complexes and spinor dynamics
}

\author{
Yaroslav V. Kartashov, ${ }^{1,2}$ Vladimir V. Konotop, ${ }^{3,4}$ and Dmitry A. Zezyulin ${ }^{3}$ \\ ${ }^{1}$ ICFO_Institut de Ciencies Fotoniques and Universitat Politecnica de Catalunya, 08860 Castelldefels, Barcelona, Spain \\ ${ }^{2}$ Institute of Spectroscopy, Russian Academy of Sciences, Troitsk, Moscow Region 142190, Russia \\ ${ }^{3}$ Centro de Física Teórica e Computacional, Faculdade de Ciências, Universidade de Lisboa, Avenida Professor Gama Pinto 2 , \\ Lisboa 1649-003, Portugal \\ ${ }^{4}$ Departamento de Física, Faculdade de Ciências, Universidade de Lisboa, Campo Grande, Edifício C8, Lisboa 1749-016, Portugal
}

(Received 22 July 2014; published 15 December 2014)

\begin{abstract}
Spin-orbit (SO) coupling can be introduced in a Bose-Einstein condensate (BEC) as a gauge potential acting only in a localized spatial domain. The effect of such a SO "defect" can be understood by transforming the system to the integrable vector model. The properties of the SO BEC change drastically if the SO defect is accompanied by the Zeeman splitting. In such a nonintegrable system, the SO defect qualitatively changes the character of soliton interactions and allows for formation of stable nearly scalar soliton complexes with almost all atoms concentrated in only one dark state. These solitons exist only if the number of particles exceeds a threshold value. We also report on the possibility of transmission and reflection of a soliton upon its scattering on the SO defect. Scattering strongly affects the pseudospin polarization and can induce pseudospin precession. The scattering can also result in almost complete atomic transfer between the dark states.
\end{abstract}

DOI: 10.1103/PhysRevA.90.063621

PACS number(s): 03.75.Lm, 03.75.Mn, 67.85.-d, 71.70.Ej

\section{INTRODUCTION}

Atomic gases in external fields represent a versatile tool for emulating phenomena originally predicted in other branches of physics, including solid-state physics [1], hydrodynamics [2], theory of gravity [3], optics [4], etc. Such systems allow for creation and control in situ of synthetic electric and magnetic fields, as well as potentials of practically any desirable shape. In this context, spin-orbit (SO) coupled BoseEinstein condensates (BECs) [5,6], experimentally realized in [7], attract particular attention as they allow for studying phenomena related to the artificial vector gauge potentials [8]. Meantime, SO BECs feature physical factors which are usually absent in the emulated systems. This is, in particular, the intrinsic nonlinearity of BECs, originating from interatomic interactions and supporting solitons in homogeneous BECs $[9,10]$ and in BECs with either Zeeman [11] or optical [12] lattices (both lattices are available experimentally $[13,14]$ ).

More features of a SO BEC can be explored due to flexibility of the SO coupling. In particular, by using an external laser beam of a finite width one can implement a localized in space SO coupling, i.e., a kind of SO coupling defect (SOD). In this situation spinor components of the macroscopic wave function are coupled to the translational motion only in a localized spatial domain and are linearly decoupled outside it. In the absence of other external fields the effect of a SOD on stationary modes consists only in imprinting of spin texture and no scattering occurs when a soliton interacts with a SOD, since the model remains integrable. A remarkable fact, however, is that the situation changes dramatically if a SOD is created in a BEC subjected to the Zeeman splitting. The system becomes nonintegrable, the character of soliton interactions changes, and unique families of essentially nonlinear modes (multipole quasiscalar complexes having no linear limit) appear. A soliton incident on the defect can be either transmitted or reflected (at weak or strong Zeeman fields, respectively), which is accompanied by precession of the pseudospin. These effects stemming from the interplay of the SOD and Zeeman splitting constitute the subject of the present paper.

The paper is organized as follows. The model is formulated in Sec. II. In Sec. III we study the bifurcation of stationary modes from the Manakov soliton solutions analytically (Sec. III A) and families of the solutions and their stability numerically (Sec. III B). In Sec. IV we describe scattering of spinor solitons on the SOD. Some technical details of calculations are given in the Appendices.

\section{THE MODEL}

We consider a cigar-shaped SO BEC elongated in the $x$ direction and tightly bounded in the $y z$ plane. The physical model for the coupling comes from the tripod scheme [15], having three $(j=1,2,3)$ ground states $|j\rangle$ and one excited state $|0\rangle$ coupled by the laser beams $\Omega_{1,2}=2^{-1 / 2} \Omega_{0} e^{-i k y \mp i K(x)} \sin \theta$ and $\Omega_{3}=\Omega e^{i k z} \cos \theta$, where $k$ is the wave vector, $\theta$ and $\Omega_{0}$ are constants characterizing the field amplitudes and phases, and $K(x)=\int_{-\infty}^{x} \kappa(\xi) d \xi$ is the phase modulation of the control beams. The characteristic size of the condensate is considered much smaller than the diffraction length of the laser beams $\Omega_{j}$, [even if $K(x)$ varies on the scale of a few microns], which allows one to neglect beam diffraction on the scale of the atomic cloud.

The linear part of the atomic Hamiltonian reads

$$
H_{\text {lin }}=-\hbar \sum_{j=1}^{3} \Omega_{j}|0\rangle\langle j|+\text { H.c. }
$$

and allows for the existence of dark states (see, e.g., [15]):

$$
\begin{array}{r}
\left|D_{1}\right\rangle=\frac{1}{\sqrt{2}} e^{i k(y+z)}\left(e^{i K(x)}|1\rangle-e^{-i K(x)}|2\rangle\right), \\
\left|D_{2}\right\rangle=\frac{\cos \theta}{\sqrt{2}} e^{i k(y+z)}\left(e^{i K(x)}|1\rangle+e^{-i K(x)}|2\rangle\right)-\sin \theta|3\rangle .
\end{array}
$$


Now the $x$ component of the synthetic vector potential $\mathbf{A}=$ $i\left\langle D_{m}(\mathbf{r}) \mid \nabla D_{n}(\mathbf{r})\right\rangle$ (i.e., the Mead-Berry connection $[15,16]$ ) is computed as $A_{x}=-\kappa(x) \sigma_{1}$ (hereafter $\sigma_{1,2,3}$ are the Pauli and $\sigma_{0}$ is the identity matrices and we use the dimensionless units defined by $m=\hbar=1$ ). Accounting for a Zeeman field $\Omega$ and for attractive two-body interactions, we describe the quasi-one-dimensional SO BEC by the spinor $\boldsymbol{\Psi}=\left(\Psi_{1}, \Psi_{2}\right)^{T}$ obeying the coupled Gross-Pitaevskii equations (GPEs) [7,17]:

$$
i \boldsymbol{\Psi}_{t}=\frac{1}{2}\left(\frac{1}{i} \frac{\partial}{\partial x}-\kappa(x) \sigma_{1}\right)^{2} \boldsymbol{\Psi}+\frac{\Omega}{2} \sigma_{3} \boldsymbol{\Psi}-\left(\boldsymbol{\Psi}^{\dagger} \boldsymbol{\Psi}\right) \boldsymbol{\Psi} .
$$

This model is exactly integrable if either Zeeman splitting or SO coupling is taken into account alone, but not both of them. If $\kappa(x) \equiv 0$, by the rotation $\boldsymbol{\Phi}=S_{\Omega}^{-1}(t) \boldsymbol{\Psi}$ with $S_{\Omega}(t)=$ $e^{-i \Omega \sigma_{3} t / 2}$, Eq. (2) is reduced to the Manakov system (MS) [18]:

$$
i \boldsymbol{\Phi}_{t}=-\frac{1}{2} \boldsymbol{\Phi}_{x x}-\left(\boldsymbol{\Phi}^{\dagger} \boldsymbol{\Phi}\right) \boldsymbol{\Phi},
$$

so that the one-soliton solution of Eq. (2) acquires the form $\boldsymbol{\Psi}=S_{\Omega}(t) \boldsymbol{\Phi}_{M}$ where

$$
\boldsymbol{\Phi}_{M}=\frac{\eta e^{i v x+i\left(\eta^{2}-v^{2}\right) t / 2}}{\cosh \left[\eta\left(x-v t-x_{0}\right)\right]}\left(\begin{array}{c}
e^{i \beta} \cos \alpha \\
e^{-i \beta} \sin \alpha
\end{array}\right),
$$

and $\eta, v, \alpha, \beta$, and $x_{0}$ are constants determining soliton parameters. If $\Omega=0$, then the MS is obtained after the spatial rotation $\boldsymbol{\Psi}=S_{\kappa}(x) \boldsymbol{\Phi}_{M}$ with

$$
S_{\kappa}(x)=\frac{1}{\sqrt{2}}\left(1-i \sigma_{2}\right) e^{i \sigma_{3} K}=\frac{1}{\sqrt{2}}\left(\begin{array}{cc}
e^{i K(x)} & -e^{-i K(x)} \\
e^{i K(x)} & e^{-i K(x)}
\end{array}\right) .
$$

If $\kappa=$ const and $\Omega=0$ (or vice versa if $\Omega=$ const and $\kappa=0$ ), then by the global rotation Eq. (2) can be rewritten in the form in which stationary localized modes were thoroughly studied in optics [19]. For the case of constant SO coupling, bright solitons were found for constant [9] and periodic [11] Zeeman fields. Those solitons had two distinguishing features: in the limit of a small number of atoms they bifurcated from the linear spectrum, and the populations of the dark states were comparable and even equal. These essentially vector solitons can form multihump complexes if repulsion between out-ofphase humps in one component is compensated by coupling with the second component.

The Zeeman field applied simultaneously with SO coupling breaks gauge symmetry, while spatial dependence of the SO coupling, $\kappa(x) \neq$ const, breaks the translational symmetry. These broken symmetries lead to much more complicated stationary and dynamical properties of the condensate. Our first main result is a new class of stationary modes having no counterparts in previously considered vector models and in the scalar nonlinear Schrödinger (NLS) model (because of repulsion or attraction between neighboring solitons [20]). These modes are (i) multisoliton complexes with no linear limit, i.e., they require a nonzero number of atoms; (ii) nearly scalar, which means that they are characterized by a large population imbalance between the spinor components (this is a counterintuitive situation as the linear coupling is supposed to act toward balancing the populations); and (iii) stable for properly chosen defect parameters.

Our second result is the peculiar interaction of a moving vector soliton with the SOD. We show almost complete transmission of a soliton through the defect at the Zeeman field below some critical value $\Omega<\Omega_{\text {cr }}$ and almost total reflection at $\Omega>\Omega_{\mathrm{cr}}$. In both cases interaction of a soliton with the defect induces the pseudospin precession.

\section{THE STATIONARY PROBLEM}

First we consider stationary modes: $\boldsymbol{\Psi}(x, t)=e^{-i \mu t} \psi(x)$, where $\mu$ is the chemical potential and $\psi(x)$ solves the stationary GPE:

$$
\mu \psi=\frac{1}{2}\left(\frac{1}{i} \frac{\partial}{\partial x}-\kappa(x) \sigma_{1}\right)^{2} \psi+\frac{\Omega}{2} \sigma_{3} \psi-\left(\psi^{\dagger} \psi\right) \psi .
$$

Even in the absence of the Zeeman splitting $(\Omega=0)$ the SOD introduces inhomogeneous spinor texture because it couples two distinct spinor states at $x= \pm \infty$. This is seen from the local Stokes components $s_{j}=\boldsymbol{\Psi}^{\dagger} \sigma_{j} \boldsymbol{\Psi}$, where $j=0, \ldots, 3$ and $\sigma_{0}$ is the identity matrix. Function $s_{0}(x, t)$ describes the density of the condensate, and $s_{1}^{2}+s_{2}^{2}+s_{3}^{2}=s_{0}^{2}$. At $\Omega=0$ one obtains from Eq. (4) that

$$
s_{1}=2 s_{0} \cos (2 \alpha), \quad s_{3}+i s_{2}=-s_{0} \sin (2 \alpha) e^{2 i(K(x)+\beta)} ;
$$

i.e., the pseudospin vector $\mathbf{s}=\left(s_{1}, s_{2}, s_{3}\right)$ changes its orientation in the $y z$ plane along the $x$ axis. The spin slope $\alpha$ and phase $\beta$ are arbitrary, so far.

\section{A. Nonlinear modes at small Zeeman field}

The situation changes when $\Omega \neq 0$. To describe this case we perform the spatial rotation $\psi=S_{\kappa}(x) \phi$ with $S_{\kappa}(x)$ defined in Eq. (5) and obtain the system for the spinor $\phi$ :

$$
\mu \phi=-\frac{1}{2} \frac{d^{2} \phi}{d x^{2}}-\frac{\Omega}{2} \sigma_{1} e^{2 i \sigma_{3} K(x)} \phi-\left(\phi^{\dagger} \phi\right) \phi .
$$

When $\Omega=0$, a stationary mode of the latter equation localized at $x=0$ is obtained from the Manakov soliton (4) at $v=x_{0}=$ 0 :

$$
\phi_{0}=\frac{\eta}{\cosh (\eta x)}\left(\begin{array}{c}
e^{i \beta} \cos \alpha \\
e^{-i \beta} \sin \alpha
\end{array}\right), \quad \eta=\sqrt{-\mu} .
$$

Let us now consider the case with $|\Omega| \ll 1$. It is convenient to introduce the dimensionless variable $\xi=\eta x$ and the spectral parameter $v=-\mu / \eta^{2}$ and to represent the wave function in the form (this representation as well as its convenience for the perturbation analysis are introduced and discussed in [22])

$$
\phi(x)=\eta e^{i \sigma_{3} \beta} S_{\alpha} \mathbf{w}(\xi), \quad S_{\alpha}=\left(\begin{array}{cc}
\cos \alpha & -\sin \alpha \\
\sin \alpha & \cos \alpha
\end{array}\right) .
$$

It is straightforward to verify that for $\Omega \neq 0$ the vector $\mathbf{w}$ solves the equation

$$
\frac{d^{2} \mathbf{w}}{d \xi^{2}}+2\left(\mathbf{w}^{\dagger} \mathbf{w}\right) \mathbf{w}-v \mathbf{w}=-\epsilon \hat{\omega}(\xi) \mathbf{w}
$$

where $\epsilon=\Omega / \eta^{2}$,

$$
\begin{aligned}
\hat{\omega}(\xi)= & \cos (2 \alpha) \cos [Q(\xi)] \sigma_{1}+\sin [Q(\xi)] \sigma_{2} \\
& +\sin (2 \alpha) \cos [Q(\xi)] \sigma_{3}
\end{aligned}
$$

and

$$
Q(\xi)=2[K(\xi / \eta)+\beta] .
$$


Next, we set $\epsilon \ll 1$ and consider the expansion

$$
\begin{aligned}
& \mathbf{w}=\mathbf{w}_{0}+\epsilon\left(\begin{array}{l}
u_{1}(\xi) \\
v_{1}(\xi)
\end{array}\right)+\epsilon^{2}\left(\begin{array}{l}
u_{2}(\xi) \\
v_{2}(\xi)
\end{array}\right)+\ldots, \\
& v=1+\epsilon v_{1}+\epsilon^{2} v_{2}+\ldots
\end{aligned}
$$

where

$$
\mathbf{w}_{0}=\frac{1}{\cosh \xi}\left(\begin{array}{l}
1 \\
0
\end{array}\right)
$$

The solvability conditions for the first-order term $\left(u_{1}, v_{1}\right)^{T}$ of this expansion yield the constraints (see Appendix A for the details)

$$
\begin{aligned}
\sin (2 \alpha) \int_{-\infty}^{\infty} \frac{\cos [Q(\xi)] \sinh (\xi) d \xi}{\cosh ^{3}(\xi)} & =0, \\
\int_{-\infty}^{\infty} \frac{\sin [Q(\xi)] d \xi}{\cosh ^{2}(\xi)} & =0, \\
\cos (2 \alpha) \int_{-\infty}^{\infty} \frac{\cos [Q(\xi)] d \xi}{\cosh ^{2}(\xi)} & =0 .
\end{aligned}
$$

Now consider the defect of the given parity, which in this section is understood as the parity of $\sin [Q(\xi)]$ and $\cos [Q(\xi)]$. Then, for the existence of a family bifurcating from the stationary Manakov solution (at $\Omega=0$ ), constraint (15a) requires $\cos [Q(\xi)]=\cos [Q(-\xi)]$, while Eq. (15b) requires $\sin [Q(-\xi)]=-\sin [Q(\xi)]$. Finally, from Eq. (15c), where the integral is nonzero, we obtain that for the bifurcation of the family the Manakov soliton must have $\alpha=(\pi / 4)+(\pi n) / 2$ ( $n$ is an integer).

Summarizing the above results we conclude that the families of solutions can bifurcate from the following Manakov solitons:

$$
\phi_{j}=\frac{\eta}{\sqrt{2} \cosh (\eta x)}\left(\begin{array}{c}
1 \\
(-1)^{j}
\end{array}\right), \quad j=1,2
$$

for an even defect $\kappa(x)=\kappa(-x)$, for which

$$
Q(\xi)=2[K(x)-K(0)]=-2[K(-x)-K(0)],
$$

provided that $\beta=-K(0)$.

Notice that for an odd defect $\kappa(x)=-\kappa(-x)$ we have that $K(x)-K(0)=K(-x)-K(0)$, and thus $Q(\xi)=Q(-\xi)$ and Eq. (15b) is generically not satisfied; i.e., there are no modes bifurcating from the Manakov soliton in the case of an odd defect.

In the original field variables, Eq. (16) means that at small $\Omega \neq 0$ branching of a nonlinear mode is only possible from the vector solitons, which at $\Omega=0$ read

$$
\psi_{1}=\frac{\sqrt{2} \eta}{\cosh (\eta x)}\left(\begin{array}{c}
\cos \{2[K(x)-K(0)]\} \\
i \sin \{2[K(x)-K(0)]\}
\end{array}\right), \quad \psi_{2}=\sigma_{1} \psi_{1}
$$

Expressions (18) reveal some features characteristic to the nonlinear modes [see Figs. 2(a) and 2(b)]: (i) there is a $\pi / 2$ phase shift between the components; (ii) the density maximum at $x=0$ in one component corresponds to the node of the other one; and (iii) a decrease of the SO coupling $(K \rightarrow 0)$ results in a scalar soliton (all atoms are concentrated in one component).

\section{B. Numerical study of the nonlinear modes}

To study the problem numerically, we focus on the Gaussian $\operatorname{SOD} \kappa(x)=(2 / \pi)^{-1 / 2}(a / w) e^{-x^{2} /\left(2 w^{2}\right)}$, where $w$ is the width of the defect and $a$ determines its amplitude. When the SO coupling and Zeeman fields are fixed, soliton families can be characterized by the dependence of the number of atoms $N=\int_{-\infty}^{\infty} s_{0}^{2}(x, t) d x$ on $\mu$ (see Fig. 1). We found that for a SOD of finite width all soliton families exist only if the number of atoms exceeds a certain critical value $N_{\text {cr. }}$. This can be understood from Eq. (2), which includes the expulsive potential $\sim \kappa^{2}(x)$ induced by the SOD. Its influence can be compensated only by sufficiently strong attractive nonlinearity $\sim \boldsymbol{\Psi}^{\dagger} \boldsymbol{\Psi}$, which requires nonzero $N$.

The found soliton families consist of the upper and lower branches joining at the cutoff value of the chemical potential $\mu_{\text {co }}$ (Figs. 1 and 3). There is an infinite set of such families with progressively increasing complexity of soliton shapes. Solitons belonging to the lower branches incorporate one, two, or more (depending on the order of the family) out-of-phase humps in the first component and have rather complex structure of the second component [Figs. 2(a), 2(e), 2(i), and 2(k)]. For solitons from the lower branch, the amplitude of the second component can be small compared to that of the first component: say in Fig. 2(e) the relation between the atomic density maxima in the states is $\left|\psi_{2}\right|^{2} /\left|\psi_{1}\right|^{2} \approx 0.010$. Therefore, these modes can be characterized as nearly scalar. Solitons from the upper branches resemble coupled monopole and dipole modes [Fig. 2(c)] for the first family, coupled dipole and tripole modes [Fig. 2(g)] for the second family, etc.

Top and bottom rows of Fig. 2 illustrate the transformation of soliton profiles upon decrease of the SO coupling strength. In Figs. 2(a) and 2(b) one observes that the second component of solitons from the lower branch nearly vanishes when $a \rightarrow 0$; i.e., one gets a conventional (nearly scalar) monopole soliton with almost all atoms concentrated in only one dark state. For $a=0$ this mode degenerates into the scalar soliton of the NLS equation and the threshold number of particles $N_{\text {cr }}$ vanishes. In contrast, the second component does not vanish for solitons from the upper branch even at $a \rightarrow 0$ [Figs. 2(c) and 2(d)]; these solitons transform into fully vectorial solitons of the MS [after the rotation $S_{\Omega}(t)$ as explained above]. The most unexpected result is shown in Figs. 2(f), 2(j), and 2(l),
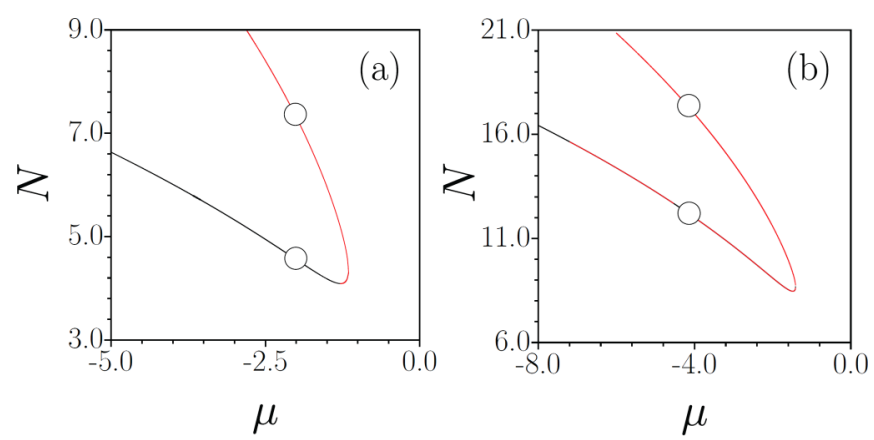

FIG. 1. (Color online) Families of monopole solitons for $w=$ 1.6 (a) and dipole solitons for $w=1.5$ (b). In all cases $a=1, \Omega=$ 1. Stable (unstable) families are shown in black (red). The circles correspond to solitons shown in Figs. 2(a), 2(c), 2(e), and 2(g). 

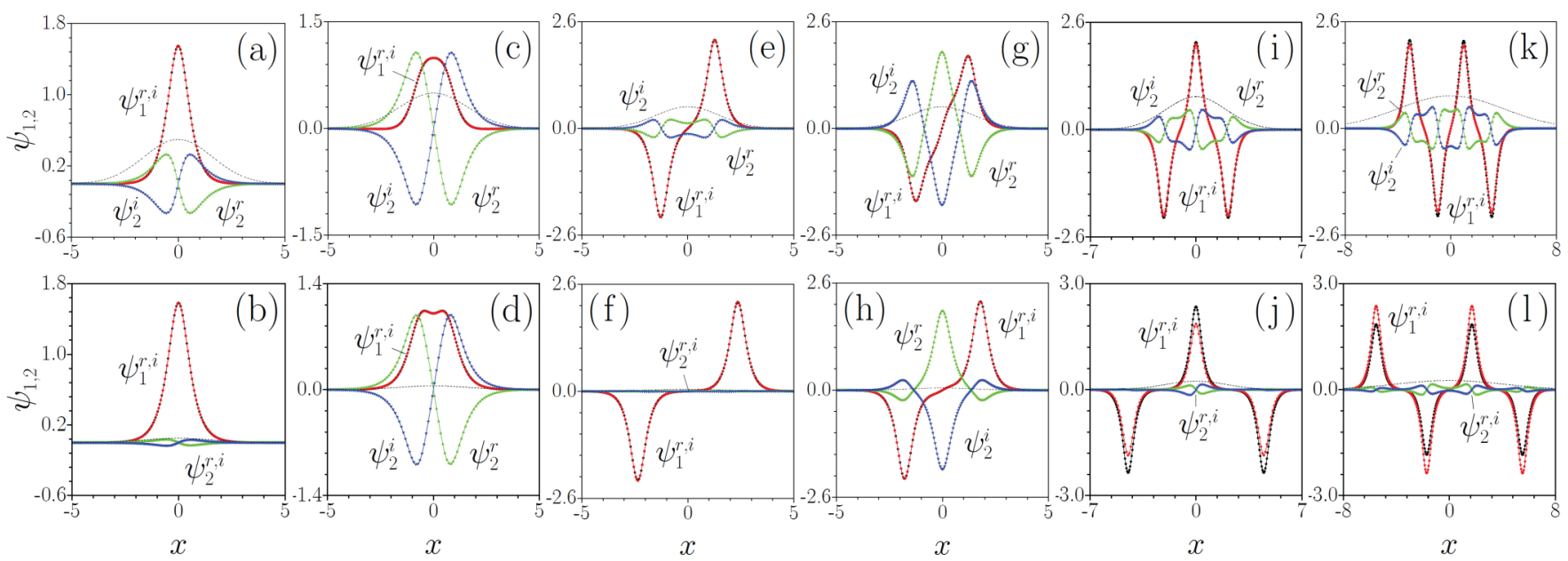

FIG. 2. (Color online) Monopole modes from lower (a, b) and upper (c, d) branches at $\mu=-2, w=1.6$ shown in Fig. 1(a). Panels (a, c) correspond to $a=1$, while panels (b, d) correspond to $a=0.1$. Dipole modes from the lower (e, $\mathrm{f}$ ) and upper (g, h) branches at $\mu=-2$, $w=1.5$ are shown in Fig. 1(b). Panels (e, g) correspond to $a=1$, while panels (f, h) correspond to $a=0.1$. Tripole solitons are from the lower branch with $\mu=-4, w=2, a=2$ (i), and $a=0.6$ (j). Quadrupole solitons are from the lower branch with $\mu=-4, w=4, a=4$ (k), and $a=1.3(1)$. In all the cases $\Omega=1$. Dashed lines show $\kappa(x)$ profiles.

illustrating that decreasing the strength of the SOD results in gradual unfolding of the multihump solitons from the lower branch into sets of well-separated nearly scalar solitons (the second component is hardly visible on the scale of Fig. 2). This means that SO coupling qualitatively changes the character of soliton interactions: it suppresses repulsion between out-of-phase humps (unavoidable in the scalar NLS equation [20]), and allows for formation of nearly scalar soliton complexes with an arbitrary number of humps.
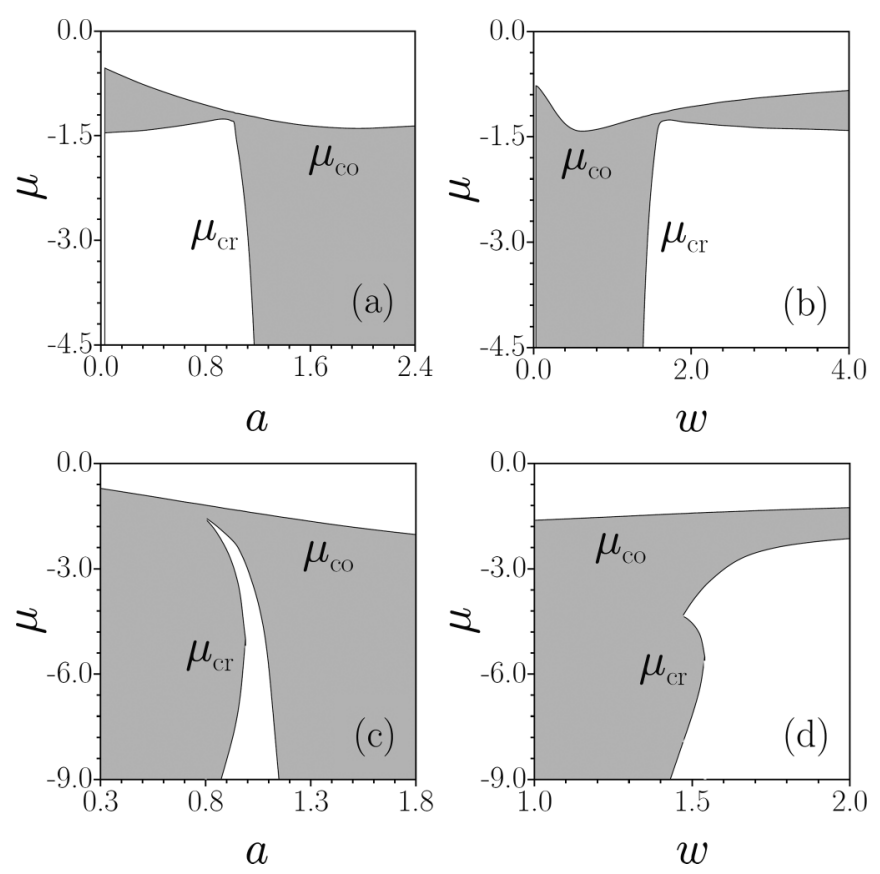

FIG. 3. Domains of stability (white) and instability (shaded) for the monopole $(\mathrm{a}, \mathrm{b})$ and dipole $(\mathrm{c}, \mathrm{d})$ solitons from the lower branches in Figs. 1(a) and 1(b), respectively. In (a,c) the defect width $w=1.6$, while in (b,d) the defect amplitude $a=1$. In all cases $\Omega=1$.
To understand qualitatively the effect of SO coupling which depends on the kinetic energy $H_{\text {kin }}=\frac{1}{2} \int \boldsymbol{\Psi}^{\dagger} p^{2} \boldsymbol{\Psi} d x$, where $p=-i \partial / \partial x$, let us consider nearly scalar modes and address the simplest case of the constant coupling $\kappa=\kappa(0)$. Assuming that $\left|\Psi_{2}\right| \ll\left|\Psi_{1}\right| \ll 1$ (i.e., the weakly nonlinear limit) and small kinetic energy, for the stationary mode we obtain $\psi_{2} \approx i \kappa \psi_{1, x} /\left(\mu+\Omega / 2+\kappa^{2} / 2\right)$. Taking into account that the phases of $\psi_{1,2}$ do not change with $x$ and differ by $\pi / 2$, we obtain an estimate for the energy of the interaction:

$$
H_{\mathrm{int}}=-i \int \kappa \psi^{\dagger} \sigma_{1} \psi_{x} d x \approx \frac{4 \kappa^{2}}{2 \mu+\Omega+\kappa^{2}} \int\left|\psi_{1 x}\right|^{2} d x
$$

For all considered modes $H_{\text {int }}<0$ and in the case if neighboring out-of-phase solitons the contribution from the term $\left|\psi_{1 x}\right|$ to the integral increases when two solitons approach each other. Therefore, the smaller is the separation between out-of-phase solitons, the smaller is $H_{\text {int }}<0$. Thus SO coupling diminishes the energy, preventing decoupling of multihump solitons. The separation between humps in soliton complexes decreases with increase of the defect amplitude $a$ [Figs. 2(e), 2(i), and $2(\mathrm{k})]$. We emphasize that these nearly scalar states do not have analogs in previously considered vector models where repulsion between out-of-phase humps in one soliton component can only be compensated at the expense of its coupling with a nearly equally strong second component.

We also examined the linear stability of the nonlinear modes (see Appendix B). We found that solitons from the upper branches are always unstable, but solitons from the lower branches can be stable in wide regions of their existence domain, presented in Fig. 3 for monopole [Figs. 3(a) and 3(b)] and dipole [Figs. 3(c) and 3(d)] solitons. For the fundamental soliton smaller defect amplitudes $a$ facilitate soliton stabilization, but the domains of stability may be rather complex for multipole solitons [Fig. 3(c)]. Stability regions are also highlighted in Fig. 1.

Nearly scalar multihump solitons exist also for a homogeneous SO coupling, which in our case corresponds to 

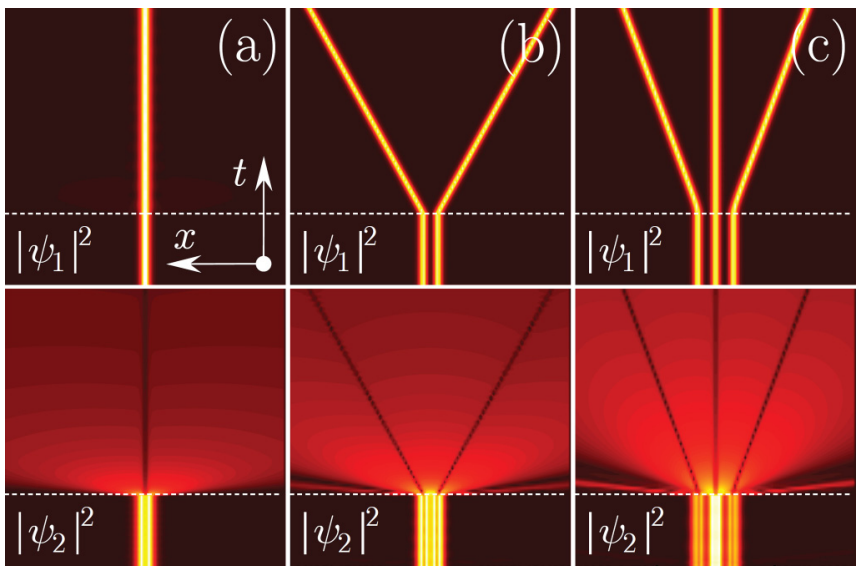

FIG. 4. (Color online) Splitting of stable modes into solitons after switching off SO coupling at $t=50$ (dashed line). The initial distributions correspond to the monopole at $\mu=-2.5, a=1, w=$ 1.6 (a); dipole at $\mu=-4.2, a=1, w=1.5$ (b); and tripole at $\mu=-4, a=1.5, w=3$ (c). In all cases $\Omega=1$ and the total evolution time is $t=200$.

$w \rightarrow \infty$ at $a / w=$ const. However, linear stability analysis have shown that all such solitons [counterparts of states in Figs. 2(e), 2(i), and 2(k)] are unstable for all $\mu$ values as long as $\kappa=$ const. This analysis was conducted by solving the associated linear eigenvalue problem. The structure of the spectrum, in particular, the presence of the eigenvalues with a positive real part indicating instability, is dictated only by the particular shape of spinor $\boldsymbol{\Psi}(x)$ and by the width of the $\kappa(x)$ function. Therefore, the finite width $w$ of the SOD is crucial, since it allows us to stabilize the solitons. The fact that SO coupling is crucial for the formation of nearly scalar multihump solitons is also illustrated in Fig. 4, where abrupt switching off the SO coupling at $t=50$ results in the unfolding of stable multihump modes into a fan of diverging scalar NLS solitons.

\section{THE SCATTERING PROBLEM}

At $x \rightarrow-\infty$ the SOD vanishes and GPEs (2) possess a solution $\boldsymbol{\Psi}=S_{\Omega}(t) \boldsymbol{\Phi}_{M}$. Now we consider interaction of this soliton moving with the initial velocity $v$ with the SOD located at $x=0$. To quantify scattering, we introduce the integral pseudospin components $S_{j}(t)=\int_{-\infty}^{\infty} s_{j}(x, t) d x, j=0, \ldots, 3$, which before collision $(x \rightarrow-\infty$, designated by superscript "_") amount to

$$
S_{1}^{-}+i S_{2}^{-}=2 \eta \sin (2 \alpha) e^{i(\Omega t-2 \beta)}, \quad S_{3}^{-}=2 \eta \cos (2 \alpha)
$$

( $S_{0}=2 \eta$ is the conserved total number of atoms). Notice that the integral components for the incident soliton satisfy the identity $\left[S_{1}^{-}\right]^{2}+\left[S_{2}^{-}\right]^{2}+\left[S_{3}^{-}\right]^{2}=S_{0}^{2}$; i.e., one can say that the incident soliton features pseudospin precession with frequency $\Omega$.

At $\Omega=0$ no pseudospin precession occurs, and the soliton also does not undergo scattering; the collision with the defect is accompanied neither by pseudospin precession nor by scattering, because the rotation $S_{\kappa}^{-1}$ reduces Eq. (2) to the MS. The situation changes in the presence of the Zeeman splitting $(\Omega>0)$, as shown in Fig. 5, where the initial solitons

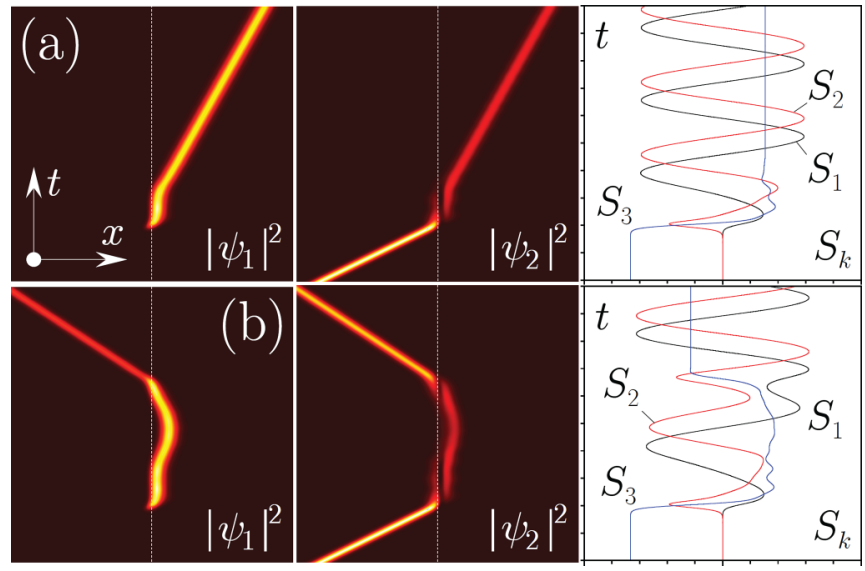

FIG. 5. (Color online) Soliton interaction with the SOD in the presence of the Zeeman splitting $\Omega=0.099$ (a) and $\Omega=0.1$ (b). The parameters of the incident soliton and the SO defect are the same in both cases: $\eta=1, \alpha=\pi / 2, v=0.4, \beta=0$, and $a=w=1$. The evolution is shown up to $t=240$ in the window $x \in[-24,24]$. The pseudospin components are shown in the window $S_{k} \in[-3,3]$.

are chosen with $\alpha=\pi / 2$ so that all atoms populate only the second state. In this case one observes either almost complete transmission through [Fig. 5 (a)] or almost complete reflection by [Fig. 5 (b)] the SOD. Although the existence of the transition region between transmission at smaller $\Omega$ and reflection at large $\Omega$ is expectable, a remarkable fact obtained numerically is a sharp transition between the two scenarios which occurs in an extremely narrow domain of variations of the Zeeman field $[\Omega=0.099$ in Fig. 5 (a) and $\Omega=0.1$ in Fig. 5(b)]. In general, the interaction scenario depends on the whole set of parameters, but reflection dominates at small velocities $(v \lesssim 0.4)$ and relatively large $\Omega$, and vice versa larger velocities (say, $v \sim 2$ ) and smaller values of $\Omega$ favor transmission.

In either of the scenarios presented in Fig. 5, the SOD induces spin precession [the third column of Fig. 5], the frequency of which is given by $\Omega$. The precession is initiated through the atom transfer between the dark states in the defect region, which changes $S_{1}$ and perturbs the initial one-soliton solution. The integrability of the system is "restored" after the soliton passes the defect, but the soliton is now transformed into a breather characterized by the internal frequency $\Omega$ (for the discussion of the two-soliton solutions of the MS see, e.g., [21]). Small modification of $\Omega$ also strongly affects the component $S_{3}(t)$, which acquires a nearly constant value after scattering [ $\sim 1$ in Fig. 5(a) and $\sim-1$ in Fig. 5(b)].

\section{CONCLUSION}

Summarizing, we introduced the system with localized SO coupling. The nontrivial interplay between SO coupling and Zeeman splitting is revealed. These two effects acting together lead to nonintegrability of the underlying GP equation and the emergence of a number of nontrivial soliton properties. The central one among them is the possibility of formation of stable quasiscalar soliton complexes, having no analogs in uniform BECs, due to qualitative modification of interaction forces between out-of-phase solitons mediated by SO coupling. The 
results obtained here may be extended to dissipative excitonpolariton BECs and to optical systems, governed by similar evolution equations.

\section{ACKNOWLEDGMENTS}

V.V.K. and D.A.Z. acknowledge support of Fundação para a Ciência e a Tecnologia (FCT, Portugal) grants PEstOE/FIS/UI0618/2014 and PTDC/FIS-OPT/1918/2012.

\section{APPENDIX A: ON BIFURCATION OF THE NONLINEAR MODES}

To perform the small amplitude expansion (13) we collect all the terms with the same powers of $\epsilon$, and obtain that in the leading order Eq. (11) is satisfied by $\mathbf{w}_{0}$ defined in Eq. (14). Proceeding in a way similar to [22] we rewrite the first-order equations in the form

$$
\hat{L} W_{1}(\xi)=F_{1}(\xi)
$$

for the vector

$$
W_{1}=\left(\begin{array}{c}
u_{1} \\
u_{1}^{*} \\
i v_{1} \\
-i v_{1}^{*}
\end{array}\right)
$$

The operator $\hat{L}$ is defined by

$$
\begin{gathered}
\hat{L}=\left(\begin{array}{cc}
L_{u} & 0 \\
0 & L_{v}
\end{array}\right), \\
L_{u}=\left(\frac{d^{2}}{d \xi^{2}}+4 u_{0}^{2}(\xi)-1\right) \sigma_{0}+2 u_{0}^{2}(\xi) \sigma_{1}, \\
L_{v}=\left(\frac{d^{2}}{d \xi^{2}}+2 u_{0}^{2}(\xi)-1\right) \sigma_{0},
\end{gathered}
$$

and the right-hand side is given by

$$
F=\frac{1}{\cosh \xi}\left(\begin{array}{c}
\sin (2 \alpha) \cos [Q(\xi)]-v_{1} / 2 \\
\sin (2 \alpha) \cos [Q(\xi)]-v_{1} / 2 \\
\cos (2 \alpha) \cos [Q(\xi)]+i \sin [Q(\xi)] \\
\cos (2 \alpha) \cos [Q(\xi)]+i \sin [Q(\xi)]
\end{array}\right)
$$

Next, defining the inner product between two-column vectors $G_{1}(\xi)$ and $G_{2}(\xi)$ by

$$
\left(G_{1}, G_{2}\right)=\int_{-\infty}^{\infty} G_{1}^{\dagger}(\xi) G_{2}(\xi) d \xi,
$$

one finds that the kernel $\hat{L}$ is spanned by four orthonormal eigenstates:

$$
\begin{aligned}
& P_{1}=\frac{1}{2 \cosh \xi}\left(\begin{array}{r}
1 \\
-1 \\
0 \\
0
\end{array}\right), \quad P_{2}=\frac{\sqrt{3} \sinh \xi}{2 \cosh ^{2} \xi}\left(\begin{array}{l}
1 \\
1 \\
0 \\
0
\end{array}\right), \\
& P_{3}=\frac{1}{\sqrt{2} \cosh \xi}\left(\begin{array}{l}
0 \\
0 \\
1 \\
0
\end{array}\right), \quad P_{4}=\frac{1}{\sqrt{2} \cosh \xi}\left(\begin{array}{l}
0 \\
0 \\
0 \\
1
\end{array}\right) .
\end{aligned}
$$

Existence of a solution of Eq. (A1) is determined by the Fredholm alternative, i.e., by the requirements $\left(P_{j}, F_{1}\right)=0$ which must be satisfied for all $j=1,2,3,4$. One readily ensures that $\left(P_{1}, F_{1}\right)=\left(P_{2}, F_{1}\right)$ and $\left(P_{3}, F_{1}\right)=\left(P_{4}, F_{1}\right)$; i.e., effectively we have two (generally speaking complex) conditions which are reduced to the conditions (15). These conditions do not involve $v_{1}$, which means that $v_{1}=0$ and hence $\mu=\mu_{0}+\mathcal{O}\left(\epsilon^{2}\right)$.

\section{APPENDIX B: ON THE LINEAR STABILITY ANALYSIS}

The linear stability analysis was performed by means of substitution of slightly perturbed wave functions $(j=1,2)$ :

$$
\Psi_{j}=\left[\psi_{j r}(x)+i \psi_{j i}(x)+\left(u_{j}+i v_{j}\right) e^{\delta t}\right] e^{-i \mu t},
$$

where the indices $r$ and $i$ stand for the real and imaginary parts and $u_{j}$ and $v_{j}$ are the real and imaginary parts of the perturbation, into GPE (2), and linearization of it with respect to $u_{j}$ and $v_{j}$, which can grow with the complex rate $\delta=\delta_{r}+i \delta_{i}$ upon evolution. This linearized eigenvalue problem reads

$$
\begin{aligned}
& \delta u_{1}=-\frac{1}{2} \frac{d^{2} v_{1}}{d x^{2}}+\frac{\kappa^{2}}{2} v_{1}+\kappa \frac{d u_{2}}{d x}+\frac{1}{2} \frac{d \kappa}{d x} u_{2}+\frac{\Omega}{2} v_{1}-\mu v_{1}-2 \psi_{1 r} \psi_{1 i} u_{1}-3 \psi_{1 i}^{2} v_{1}-2 \psi_{1 i} \psi_{2 r} u_{2}-2 \psi_{1 i} \psi_{2 i} v_{2}-\left(\left|\psi_{2}\right|^{2}+\psi_{1 r}^{2}\right) v_{1}, \\
& \delta v_{1}=\frac{1}{2} \frac{d^{2} u_{1}}{d x^{2}}-\frac{\kappa^{2}}{2} u_{1}+\kappa \frac{d v_{2}}{d x}+\frac{1}{2} \frac{d \kappa}{d x} v_{2}-\frac{\Omega}{2} u_{1}+\mu u_{1}+2 \psi_{1 r} \psi_{1 i} v_{1}+3 \psi_{1 r}^{2} u_{1}+2 \psi_{1 r} \psi_{2 r} u_{2}+2 \psi_{1 r} \psi_{2 i} v_{2}+\left(\left|\psi_{1}\right|^{2}+\psi_{2 i}^{2}\right) u_{1}, \\
& \delta u_{2}=-\frac{1}{2} \frac{d^{2} v_{2}}{d x^{2}}+\frac{\kappa^{2}}{2} v_{2}+\kappa \frac{d u_{1}}{d x}+\frac{1}{2} \frac{d \kappa}{d x} u_{1}-\frac{\Omega}{2} v_{2}-\mu v_{2}-2 \psi_{2 r} \psi_{2 i} u_{2}-3 \psi_{2 r}^{2} v_{2}-2 \psi_{2 i} \psi_{1 r} u_{1}-2 \psi_{2 i} \psi_{1 i} v_{1}-\left(\left|\psi_{1}\right|^{2}+\psi_{2 r}^{2}\right) v_{2}, \\
& \delta v_{2}=\frac{1}{2} \frac{d^{2} u_{2}}{d x^{2}}-\frac{\kappa^{2}}{2} u_{2}+\kappa \frac{d v_{1}}{d x}+\frac{1}{2} \frac{d \kappa}{d x} v_{1}+\frac{\Omega}{2} u_{2}+\mu u_{2}+2 \psi_{2 r} \psi_{2 i} v_{2}+3 \psi_{2 r}^{2} u_{2}+2 \psi_{2 r} \psi_{1 r} u_{1}+2 \psi_{2 r} \psi_{1 i} v_{1}+\left(\left|\psi_{1}\right|^{2}+\psi_{2 i}^{2}\right) u_{2}
\end{aligned}
$$

It was solved numerically in order to get the dependence of the perturbation growth rate $\delta$ on the chemical potential $\mu$. The solitons are stable as long as $\delta_{r} \leqslant 0$. 
[1] M. Lewenstein, A. Sanpera, V. Ahufinger, B. Damski, B. A. Sende, and U. Sen, Adv. Phys. 56, 243 (2007).

[2] C. Pethick and H. Smith, Bose-Einstein Condensation in Dilute Gases (Cambridge University Press: Cambridge, 2002); L. P. Pitaevskii and S. Stringari, Bose-Einstein Condensation (Clarendon Press: Oxford and New York, 2003).

[3] L. J. Garay, J. R. Anglin, J. I. Cirac, and P. Zoller, Phys. Rev. Lett. 85, 4643 (2000); C. Barceló, S. Liberati, and M. Visser, Int. J. Mod. Phys. A 18, 3735 (2003).

[4] Y. V. Kartashov, B. A. Malomed, and L. Torner, Rev. Mod. Phys. 83, 247 (2011).

[5] V. Galitski and I. B. Spielman, Nature (London) 494, 49 (2013).

[6] T. D. Stanescu, B. Anderson, and V. Galitski, Phys. Rev. A 78, 023616 (2008).

[7] Y. J. Lin, K. Jimenez-Garcia, and I. B. Spielman, Nature (London) 471, 83 (2011).

[8] J. Dalibard, F. Gerbier, G. Juzeliūnas, and P. Öhberg, Rev. Mod. Phys. 83, 1523 (2011).

[9] V. Achilleos, D. J. Frantzeskakis, P. G. Kevrekidis, and D. E. Pelinovsky, Phys. Rev. Lett. 110, 264101 (2013).

[10] Y. Xu, Y. Zhang, and B. Wu, Phys. Rev. A 87, 013614 (2013).

[11] Y. V. Kartashov, V. V. Konotop, and F. K. Abdullaev, Phys. Rev. Lett. 111, 060402 (2013).

[12] H. Sakaguchi and B. Li, Phys. Rev. A 87, 015602 (2013); Y. Zhang and C. Zhang, ibid. 87, 023611 (2013).

[13] K. Jiménez-García, L. J. LeBlanc, R. A. Williams, M. C. Beeler, A. R. Perry, and I. B. Spielman, Phys. Rev. Lett. 108, 225303 (2012).
[14] C. Hamner, Yongping Zhang, M. A. Khamehchi, Matthew J. Davis, and P. Engels, arXiv:1405.4048 [cond-mat.quant-gas].

[15] J. Ruseckas, G. Juzeliūnas, P. Öhberg, and M. Fleischhauer, Phys. Rev. Lett. 95, 010404 (2005); G. Juzeliūnas, J. Ruseckas, M. Lindberg, L. Santos, and P. Öhberg, Phys. Rev. A 77, 011802 (2008).

[16] M. V. Berry, Proc. R. Soc. London, Ser. A 392, 45 (1984); F. Wilczek and A. Zee, Phys. Rev. Lett. 52, 2111 (1984); C. A. Mead, Rev. Mod. Phys. 64, 51 (1992).

[17] Y. Zhang, Li Mao, and C. Zhang, Phys. Rev. Lett. 108, 035302 (2012).

[18] S. V. Manakov, Zh. Eksp. Teor. Fiz. 67, 543 (1974) [Sov. Phys. JETP 38, 248 (1974)].

[19] D. N. Christodoulides and R. I. Joseph, Opt. Lett. 13, 53 (1988); B. A. Malomed, Phys. Rev. A 43, 410 (1991); V. M. Eleonskii, V. G. Korolev, N. E. Kulagin, and L. P. Shil'nikov, Zh. Eksp. Teor. Fiz. 99, 1113 (1991) [Sov. Phys. JETP 72, 619 (1991)]; M. Haelterman and A. Sheppard, Phys. Rev. E 49, 3376, (1994); De Angelis and S. Wabnitz, Opt. Commun. 125, 186 (1996).

[20] J. Yang, Nonlinear Waves in Integrable and Nonintegrable Systems (SIAM, Philadelphia, 2010).

[21] R. Radhakrishnan, M. Lakshmanan, and J. Hietarinta, Phys. Rev. E 56, 2213 (1997); J. Yang and D. J. Benney, Stud. Appl. Math. 96, 111 (1996).

[22] T. I. Lakoba and D. J. Kaup, Phys. Rev. E 56, 6147 (1997). 